\title{
Association algorithm to mine the rules that govern enzyme definition and to classify protein sequences Shih-Hau Chiu ${ }^{1,2}$, Chien-Chi Chen ${ }^{1}$, Gwo-Fang Yuan ${ }^{1}$ and Thy-Hou Lin*2
} Address: ${ }^{1}$ Bioresource Collection and Research Center, Food Industry Research and Development Institute, HsinChu, Taiwan and ${ }^{2}$ Institute of
Molecular Medicine/Department of Life Science, National Tsing Hua University, HsinChu, Taiwan

Email: Shih-Hau Chiu - shc@firdi.org.tw; Chien-Chi Chen - chj@firdi.org.tw; Gwo-Fang Yuan - gfy@firdi.org.tw; ThyHou Lin* - thlin@life.nthu.edu.tw

* Corresponding author

Published: 15 June 2006

BMC Bioinformatics 2006, 7:304 doi:10.1/86/147|-2105-7-304
Received: 06 December 2005

Accepted: 15 June 2006

This article is available from: http://www.biomedcentral.com/|47|-2/05/7/304

(c) 2006 Chiu et al; licensee BioMed Central Ltd.

This is an Open Access article distributed under the terms of the Creative Commons Attribution License (http://creativecommons.org/licenses/by/2.0), which permits unrestricted use, distribution, and reproduction in any medium, provided the original work is properly cited.

\begin{abstract}
Background: The number of sequences compiled in many genome projects is growing exponentially, but most of them have not been characterized experimentally. An automatic annotation scheme must be in an urgent need to reduce the gap between the amount of new sequences produced and reliable functional annotation. This work proposes rules for automatically classifying the fungus genes. The approach involves elucidating the enzyme classifying rule that is hidden in UniProt protein knowledgebase and then applying it for classification. The association algorithm, Apriori, is utilized to mine the relationship between the enzyme class and significant InterPro entries. The candidate rules are evaluated for their classificatory capacity.
\end{abstract}

Results: There were five datasets collected from the Swiss-Prot for establishing the annotation rules. These were treated as the training sets. The TrEMBL entries were treated as the testing set. A correct enzyme classification rate of $70 \%$ was obtained for the prokaryote datasets and a similar rate of about $80 \%$ was obtained for the eukaryote datasets. The fungus training dataset which lacks an enzyme class description was also used to evaluate the fungus candidate rules. A total of 88 out of 5085 test entries were matched with the fungus rule set. These were otherwise poorly annotated using their functional descriptions.

Conclusion: The feasibility of using the method presented here to classify enzyme classes based on the enzyme domain rules is evident. The rules may be also employed by the protein annotators in manual annotation or implemented in an automatic annotation flowchart.

\section{Background}

The number of sequences generated by many genome projects is soaring exponentially but most of them have not been characterized experimentally. Manual annotation methods have been proposed by experts and are popular for use at the genome centers, but their annotation capacities are exceeded by the fast growing genome data. An automatic annotation scheme is in urgent need to speed up reliable functional annotation on new sequences produced. Automatic annotation provides an efficient procedure for analyzing the gene sequences. Most automatic solutions used to characterize the gene sequences are based on a high-level sequence similarity search against some known protein databases such as using the BLAST or FASTA program. The correlation between sequence composition and functional character- 
ization provides the foundation for transferring functional knowledge from a biochemically characterized protein to a homologous but uncharacterized one. However, sequence composition bias and database updating commonly influence the results of similarity searches, and they do not yield the exact share between biological function and domain composition based on the similarity threshold used [1]. Many annotation packages have recently been developed. For example, a basic annotation, which is directly transferred from a homologue entry using a similarity threshold, is offered by the package GeneQuiz [2]. Other packages, such as Rulebase [3] and HAMAP [1], use the classifying rules and are supported by the judgment of a curator.

In the post-genomic era, the functional annotations are of great importance in understanding the real cellular processes. A variety of enzymes and pathway databases including Ecocyc, Enzyme, and KEGG, have been built to facilitate the prediction of the metabolic pathway. Such databases are supplied as reference databases in virtual construction of the metabolic networks of other organisms. On the pathway map, enzymes are the main components used for linking the metabolic networks. The fundamental units of enzyme structure governing folding and function are domains of protein [4]. A domain is believed to be able to fold independently into a stable three-dimensional structure to perform a specific function. In general, a protein would comprise a single domain or several different domains. It is clear that the domain composition of a protein determines its function and pathways in which it participates [5]. In other words, the protein function may be inferred from the domain composition which is then used to annotate the unknown sequences sharing the same domain composition with the protein. More importantly, such rules are invariable unlike the BLAST results that typically vary as the database is updated. Many tools are available for detecting the constituents of proteins such as CDART (NCBI) and InterProScan. InterPro is a database of protein families, domains, and functional sites where identifiable characteristics of known proteins can be applied to annotate unknown protein sequences [6]. The tools of InterPro, InterProScan, can be also used to annotate the single domain protein sequence; however, it may be difficult to make a decision on the annotation of multi-domain proteins by the method.

This work proposes a machine learning method for identifying enzyme classes according to the rules that are related to the protein domain composition. Using rules generated by machine learning algorithms, Kretschmann et al. [7] and Bazzan et al. [8] have successfully annotated the Trembl database. They adopted the decision tree algorithm to obtain rules from the Swiss-Prot entries that are cross-referenced to the InterPro database. They then used these rules to assign appropriate keywords to the TrEMBL entries $[7,8]$. In this study, an association algorithm is used to mine the rules linking enzymes and domains and they are then used to annotate enzyme classes automatically. The association algorithm has been extensively employed to analyze market baskets. It is applied primarily to determine the relationships among items in a large dataset. In market basket analysis, large associated itemsets always represent items that are likely to be purchased together by customers in a single transaction. The association algorithm has also been employed to mine gene expression data [9] and medical data [10]. This investigation utilizes an association algorithm to mine the rules linking enzymes and domains from the Swiss-Prot protein knowledgebase. The enzyme class and InterPro accession number (henceforth IPR Acc's) are treated consistently as items in searching for rules governing the enzyme domain composition. These rules may be useful even for annotators who do not have deep knowledge on the definitions of enzyme classes.

\section{Results and discussion \\ Data preparation}

This work seeks to annotate unknown genes and establishes virtual metabolic pathways using the bioinformatics approach based on progress made in the Monascus genome project at the authors' institute. Only few Monascus genes have been biochemically characterized so far. Numerous well-characterized proteins have been stored in a public database so that it is feasible to mine the classified rules from a protein knowledgebase. The BLAST is a fast but insufficient method for annotating unknown genes because it does not provide information on the functional domain. Analyzing the constituent domains of a gene enables the determination of possible functions of the gene. However, making a decision regarding the annotation of a multi-domain protein is difficult. In this study, an annotation model was established by applying rules derived from the domain compositions in some wellcharacterized proteins. The concept of annotation using the domain composition was further investigated. Five datasets (Table 1) were used to mine the association rules, which were then evaluated. All the datasets used herein have the EC class and IPR description. In the preliminary investigation, all the IPR Accs of each Swiss-Prot entry were utilized to determine the association rules. Unfortunately, some IPR Accs were presented as a single rule whose entries were linked to approximate sequence position but assigned with different accession numbers. To reduce the redundant and insignificant ones such as the glycosylation site and others, the IDA in InterPro was employed to filter the IPR entries. 
Table I: Five distinctly taxonomic datasets referring to the NEWT were used for generating and evaluating rules.

\begin{tabular}{cccccc}
\hline & A & B & C & D & E \\
\hline Training Instances & 3251 & 7522 & 3666 & 1791 & 4502 \\
Training Attributes & 657 & 784 & 823 & 589 & 551 \\
Testing Instances & 3440 & 10226 & 1759 & 1551 & 5022 \\
Testing Attributes & 777 & 1054 & 491 & 212 & 507 \\
\hline
\end{tabular}

A: actinobacteria B: bacillales C: fungi D: nematode + arthropoda E: viridiplantae

Association algorithm used to mine enzyme composition Many data mining methods have been applied in the biological researches. For example, a decision tree has been used in keyword annotation in the Swiss-Prot [7] and PIGS [8] projects. Herein, the association algorithm was employed to find rules to perform automatic annotation. The association algorithm has been extensively used to elucidate the consumptive behavior. These rules are ordinarily mined from numerous transaction records. Similar to the market basket analysis, the EC class and IPR Acc's were treated as a single transaction record in every training entry. In the training file (Fig. 1), each instance was composed of all the attributes (IPR Acc's) of the training dataset and all the EC class was included in the target class. The results indicated that various rules were obtained simultaneously. The candidate association rules were found redundant and many were subsets of larger frequent itemsets. Table 2 presented the subset of fungous association rules thus obtained. The complete set of rules was shown in the additional file [see Additional file 1]. The rules revealed, for instance, when the InterProScan results of the protein sequence gave IPR000873, IPR001031, IPR001242, and IPR006163, the protein was identified as EC 6.3.2.26. Table 3 listed the association rules obtained from the five datasets. About $40 \sim 70 \%$ of the rules thus obtained were the multiple domain rules ( $>=2$ domains). Although the single domain rules dominate some datasets, the multiple domain rules are more important in the annotation tasks.

\section{Evaluation of candidate rules}

As presented in Table 4, the testing dataset from TrEMBL was used to evaluate the candidate rules. The precision was around $70 \%$ for the prokaryote dataset (A and B) though the coverage was less than $50 \%$. The precision and coverage for the eukaryote datasets ( $C, D$ and $E$ ) were better than those for the prokaryote ones. The prokaryote training dataset appears to be more diversified than the eukaryote one which results in the number of rules obtained for the former was less than that for the latter. Additionally, the prediction coverage was enhanced substantially while there were redundancies remaining in the candidate rules. In fact, the rules from the subsets of the large itemset were used to predict entries that were not exactly matched with the rules from the large itemset though the prediction accuracy was slightly decreased.

Table 5 displays the cross evaluation results for the five datasets. Both precision and confidence estimated from the cross evaluation on various phylogenetic datasets (such as using a fungus testing dataset to evaluate the plant rules) were worse than those estimated on the same taxonomic dataset. This reveals that the accuracy of the prediction depends on the taxonomic relationship between the training and testing datasets. The closer the taxonomic relationship between datasets used the greater the predictive capacity obtained. Moreover, we found that there was at least $40 \%$ accuracy in different taxonomic cross-validation. It seems that some domain compositions of enzyme were similar among different taxonomic dataset. Additionally, the prediction accuracy may reflect the taxonomic relationship in the different dataset. Yang et al. [11] proposed that using only the presence or absent of a protein domain architecture can determinate the phylogeny of 174 complete genomes. Our results also reveal

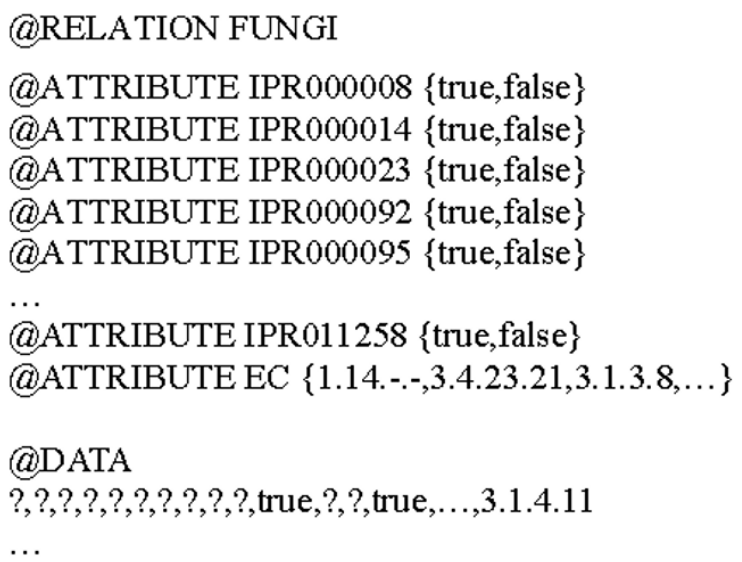
replaced with a "?" mark as a msising datum to prevent the generation of useless association rules. 
Table 2: Subset of rules generated from the fungus training dataset. The complete set of rules was shown in the additional file [see Additional file I].

\begin{tabular}{|c|c|}
\hline Association rules & EC ID \\
\hline IPR000873,IPR00 I03 I,IPR00 I 242,IPR006 I 63 & 6.3.2.26 \\
\hline IPR00I03I,IPR00|242,IPR006I63 & 6.3.2.26 \\
\hline IPR000873,IPR00103 I,IPR006I63 & 6.3.2.26 \\
\hline IPR000873,IPR00 I03 I,IPR00I 242 & 6.3.2.26 \\
\hline IPR0023|4,IPR0023I7 & 6.1.1.11 \\
\hline IPR001926,IPR002028 & 4.2.1.20 \\
\hline IPR00I03I,IPR00I 242 & 6.3.2.26 \\
\hline IPR000873,IPR00 I03। & 6.3.2.26 \\
\hline IPR000850,IPR007862 & 2.7.4.3 \\
\hline IPR007862 & 2.7.4.3 \\
\hline IPR004308 & 6.3.2.2 \\
\hline IPR003I7I & I.5.1.20 \\
\hline IPR002934 & 2.7.7.19 \\
\hline IPR000873,IPR00 I 03 I,IPR00 I 242,IPR006I 64 & 6.3.2.26 \\
\hline IPR00103I,IPR00I242,IPR006I64 & 6.3.2.26 \\
\hline IPR000873,IPR00 I03 I,IPR003679 & 6.3.2.26 \\
\hline IPR000873,IPR00 I03I,IPR008600 & 6.3.2.26 \\
\hline IPR0023 |4,IPR0023 I8 & 6.1.1.12 \\
\hline IPR001926,IPR002029 & 4.2.1.21 \\
\hline IPR00I03I,IPR00I 243 & 6.3.2.26 \\
\hline IPR000873,IPR00I032 & 6.3.2.26 \\
\hline IPR000850,IPR007863 & 2.7.4.3 \\
\hline IPR007862 & 2.7.4.3 \\
\hline IPR004308 & 6.3.2.3 \\
\hline IPR000873,IPR00 I03 I,IPR00 I 242,IPR006I64 & 6.3.2.26 \\
\hline IPR00103I,IPR00I242,IPR006I64 & 6.3.2.26 \\
\hline IPR000873,IPR00 I03 I,IPR003679 & 6.3.2.26 \\
\hline IPR000873,IPR00 I03I,IPR008600 & 6.3.2.26 \\
\hline IPR0023 I4,IPR0023 I8 & 6.1.1.12 \\
\hline IPR001 926,IPR002029 & 4.2.1.21 \\
\hline
\end{tabular}

that domain composition of enzyme is highly similar in the same taxonomic clade.

Furthermore, the accuracy of the presented method was compared with the rules obtained from the InterPro database. These rules were parsed where the IPR Acc's were cross-referenced to ENZYME in the entry_xref table of the InterPro database. The rules such as \{IPR001711, EC 3.1.4.11\} were retained for providing the enzyme identification. There were five testing datasets used to evaluate the parsed ones. As shown in Table 6, the identification accuracy was below $65 \%$. The results revealed that it was not suitable to directly parse the cross-reference between enzyme and InterPro Acc's without classifying the dataset beforehand. In other words, as mentioned above, the identification of enzyme classes should use the closer taxonomic rules. Moreover, the rules generated from the association algorithm were highly specific in the closer taxonomic testing dataset. The association algorithm was able to select more confident rules in the protein database. As shown in Table 7 and 8, our single domain rules can identify enzyme classes with high accuracy, while multiple domain rules can lift the hit ratio in the enzyme identification. In addition, the remaining datasets which were not annotated with an EC class in the fungus dataset of Swiss-Prot entries were further employed to evaluate the fungus rule set. A total of 88 out of 5085 test entries were found to match with the fungus rule set (Table 9). Most of these were otherwise poorly annotated by their functional description. These indicate that the rules mined from the association algorithm were unique to the enzyme class and could be used to annotate some unknown protein sequences.

The precision and confidence of each EC class was also evaluated in the fungus dataset. Both quantities were varied among all the EC classes tested (data not shown here). However, a precision of greater than $75 \%$ was obtained for $60 \%$ of the EC classes tested (data not shown.). In this study, the Swiss-Prot entries were chosen as the training while the TrEMBL entries as the test set. We aimed to find the EC classifying rules that are hidden in the protein knowledgebase and to estimate the accuracy of the classifying method. The rules mined and presented here can be used by an annotator to perform manual annotation. They can be also implemented in an automatic annotation flowchart. They are also feasible to be used in identifying enzyme classes based on their IPR signature.

\section{Conclusion}

This report proposed an alternative approach on employing the association algorithm. The association algorithm is commonly used to identify large and frequent item sets and mine hidden relationships among items. The concept can be applied in many fields other than market basket analysis. The method is extended here to mine the association rules which are then applied to identify enzyme classes. The current prediction scheme emphasizes on identifying enzymes of taxonomically closed datasets.

Table 3: Number of rules and classified EC generated from the training dataset.

\begin{tabular}{cccccc}
\hline & A & B & C & D & E \\
\hline Rules & 624 & 607 & 920 & 1096 & 428 \\
EC & 254 & 229 & 167 & 168 & 153 \\
multiple domain rule & $40 \%$ & $43 \%$ & $69 \%$ & $72 \%$ & $42 \%$ \\
\hline
\end{tabular}

A: actinobacteria B: bacillales C: fungi D: nematode + arthropoda E: viridiplantae 
Table 4: Evaluation of the generated candidate rules. The testing dataset was used to validate the corresponding set of rules. For instance, the fungus testing dataset was used to evaluate the set of rules generated from the fungus training dataset.

\begin{tabular}{llllll}
\hline & A & B & C & D & E \\
\hline precision & $71 \%$ & $76 \%$ & $87 \%$ & $88 \%$ & $77 \%$ \\
confidence & $69 \%$ & $74 \%$ & $85 \%$ & $85 \%$ & $75 \%$ \\
coverage* & $43 \%$ & $38 \%$ & $60 \%$ & $54 \%$ & $56 \%$ \\
\hline
\end{tabular}

A: actinobacteria B: bacillales C: fungi D: nematode + arthropoda E: viridiplantae

*: coverage $=$ the hit ratio of testing data

Rule sets generated from the eukaryote training datasets can be used to assign the EC classes accurately to poorly annotated entries whose real enzyme function remain unknown. Extending the method to predict other types of data, including the transcription factors and structure proteins, is also worthwhile. However, the low coverage is a shortcoming of the presented scheme. The matching coverage depends on the quality of the training dataset which may be extended as a combination of various datasets with each being closed in taxonomic relationship. Moreover, more rules may be generated using other association algorithms except the Apriori one.

\section{Methods}

\section{Data preparation}

There were five distinctly taxonomic datasets referring to the NEWT [12] (UniProt 4.1) being downloaded (Table 1). The entries that have multiple EC description numbers were ignored. The training datasets were the Swiss-Prot entries while the test datasets were taken from TrEMBL. The EC numbers corresponding to the Swiss-Prot entries were parsed from the field 'Description' in the Swiss-Prot database. The InterPro entries that were relevant to the Swiss-Prot entries were extracted from the InterPro database (release 9.0). The extracted data were stored in a MySQL database. The IDA (InterPro Domain Architecture) definition was also extracted from the InterPro database. Not all of the InterPro entries that corresponded to the UniProt entries were treated as the training or testing attributes. The redundant and insignificant InterPro entries were removed based on the IDA definition. The redundancy-deprived data were also stored in the MySQL database.

\section{Appling association rules determine potential enzyme composition}

The WEKA machine learning package [13] which is a freeware issued under the GNC General Public License was used to mine the association rules. The enzyme class and IPR Accs were consistently treated as items in searching for rules that governing the enzyme composition. For example, \{IPR002019, IPR002026, IPR006680, IPR011612 and EC 3.5.1.5\} in O00084 were considered to be a single transaction in the context of market basket analysis. The data stored in the MySQL database were transformed into the WEKA format (Fig. 1). The first line indicates which dataset was analyzed. (In this case, the file refers to the fungi training dataset.). The 822 lines (each headed with '@ATTRIBUTE IPR') that followed were all individual IPR Accs in the fungi training dataset. All the attributes were specified only by the value of 'true' or 'false' to indicate whether or not they were related with the given Swiss-Prot entry. The last attribute (labeled with '@ATTRIBUTE EC') was the classified target or class that consisted of all the unique EC classes in the training dataset. Finally, the 3666 lines that were behind the '@DATA' label were all the Swiss-Prot entries in the fungi training dataset used. Each of these comprises 823 entries and was separated by a comma. The interior 'false' value was replaced by a question mark to avoid the meaningless rules.

The Apriori $[14,15]$ module in the WEKA package, implemented on a linux workstation, was employed to scan the frequent itemsets and determine the associative relationships. The association rule model represents rules where a set of items was associated with each other. For instance, a rule could specify a certain product that was frequently

Table 5: Cross validation of the rule sets. The fungus testing dataset was used to evaluate the rule sets generated from the A, B, C, D, and $E$ training datasets.

\begin{tabular}{|c|c|c|c|c|c|c|c|c|c|c|}
\hline & \multicolumn{2}{|c|}{ A rule set } & \multicolumn{2}{|c|}{$B$ rule set } & \multicolumn{2}{|c|}{ C rule set } & \multicolumn{2}{|c|}{$\mathrm{D}$ rule set } & \multicolumn{2}{|c|}{ E rule set } \\
\hline & precision & confidence & precision & confidence & precision & confidence & precision & confidence & precision & confidence \\
\hline A testing data & $71 \%$ & $69 \%$ & $72 \%$ & $69 \%$ & $59 \%$ & $56 \%$ & $46 \%$ & $42 \%$ & $52 \%$ & $48 \%$ \\
\hline$B$ testing data & $68 \%$ & $67 \%$ & $76 \%$ & $74 \%$ & $61 \%$ & $59 \%$ & $43 \%$ & $41 \%$ & $51 \%$ & $49 \%$ \\
\hline$C$ testing data & $72 \%$ & $68 \%$ & $69 \%$ & $65 \%$ & $87 \%$ & $85 \%$ & $79 \%$ & $76 \%$ & $66 \%$ & $62 \%$ \\
\hline D testing data & $66 \%$ & $61 \%$ & $43 \%$ & $38 \%$ & $74 \%$ & $72 \%$ & $88 \%$ & $85 \%$ & $68 \%$ & $65 \%$ \\
\hline E testing data & $52 \%$ & $50 \%$ & $65 \%$ & $62 \%$ & $60 \%$ & $58 \%$ & $64 \%$ & $62 \%$ & $77 \%$ & $75 \%$ \\
\hline
\end{tabular}

A: actinobacteria B: bacillales C: fungi D: nematode + arthropoda E: viridiplantae 
Table 6: The five datasets used to evaluate the rules parsed from the InterPro database.

\begin{tabular}{cccc}
\hline & precision & $\begin{array}{c}\text { the parsed rules } \\
\text { Confidence }\end{array}$ & coverage* \\
\hline A testing data & $52 \%$ & $48 \%$ & $23 \%$ \\
B testing data & $51 \%$ & $50 \%$ & $24 \%$ \\
C testing data & $56 \%$ & $52 \%$ & $26 \%$ \\
D testing data & $47 \%$ & $41 \%$ & $20 \%$ \\
E testing data & $64 \%$ & $62 \%$ & $47 \%$ \\
\hline
\end{tabular}

A: actinobacteria B: bacillales C: fungi D: nematode + arthropoda E: viridiplantae

*: coverage $=$ the hit ratio of testing data

\#: The dataset was parsed from the entry xref table of the InterPro database. The IPR Acc's were corresponding to ENZYME.

Table 7: Accuracy of the single domain rules divided from the five rule sets.

\begin{tabular}{llllll}
\hline & A & B & C & D & E \\
\hline precision & $68 \%$ & $71 \%$ & $87 \%$ & $85 \%$ & $77 \%$ \\
confidence & $65 \%$ & $70 \%$ & $85 \%$ & $82 \%$ & $75 \%$ \\
coverage* & $31 \%$ & $28 \%$ & $48 \%$ & $41 \%$ & $46 \%$ \\
\hline
\end{tabular}

A: actinobacteria B: bacillales C: fungi D: nematode + arthropoda E: viridiplantae

$*$ : coverage $=$ the hit ratio of testing data

Table 8: Accuracy of the multiple domain rules divided from the five rule sets.

\begin{tabular}{lccccc}
\hline & A & B & C & D & E \\
\hline precision & $79 \%$ & $87 \%$ & $87 \%$ & $97 \%$ & $76 \%$ \\
confidence & $75 \%$ & $85 \%$ & $82 \%$ & $93 \%$ & $72 \%$ \\
coverage* & $12 \%$ & $10 \%$ & $11 \%$ & $13 \%$ & $10 \%$ \\
\hline
\end{tabular}

A: actinobacteria B: bacillales C: fungi D: nematode + arthropoda E: viridiplantae

*: coverage $=$ the hit ratio of testing data

bought in combination with other products. The rules were extracted from some large and frequently occurring itemsets. An itemset was regarded as frequent if the possibility of its occurrence exceeded a specified minimal support criterion. The algorithm proceeds iteratively to identify the frequent itemsets consisting of a single item. Then, the identified frequent itemsets were expanded with one more item to generate larger frequent itemsets. After all the frequent itemsets were identified, the candidate rules were screened through the following 'lift' criterion.

Support $(\mathrm{AB})=P(A+B)$

Confidence $(\mathrm{AB})=P(B \mid A)$

$$
\operatorname{lift}(\mathrm{AB})=\frac{P(B \mid A)}{P(B)}
$$

where $P(B \mid A)$ was the conditional probability of $B$ given $A$, and $P(A)$ or $P(B)$ was the probability of $A$ or $B$ over all instances. The probability was defined as the observed fre- quency in the data set. The support of the rule was the relative frequency of transactions containing both $\mathrm{A}$ and $\mathrm{B}$. The lift was the related measure of strength of the association. Positive correlation was indicated by lift $>1$ while negative correlation was indicated by lift $<1$. A large frequent itemsets were subdivided into smaller ones in numerous ways to generate the candidate association rules. The candidate association rules were redundant and many of them were subsets of larger frequent itemsets. However, the rules mined herein were of the form $\{A, B, C\} \Rightarrow\{D\}$ but not $\{A, B\} \Rightarrow\{C, D\}$ or $\{A\} \Rightarrow\{B, C, D\}$. For example, $\{$ IPR000873, IPR006163, IPR010080 $\} \Rightarrow$ $\{1.2 .1 .31\}$. Because most of the support values of items were between 1 and 50, a minimum support value of $0.09 \%$ was set herein to indicate that the attribute must appear 2.7 times in 3000 instances. The threshold of confidence was 0.6 and the corresponding lift value was between 10 and 30 . 
Table 9: Examples of the matching entries which were not annotated with an EC class in the remaining fungus dataset of Swiss-Prot entries were predicted using the fungus rule set.

\begin{tabular}{|c|c|c|c|}
\hline Swiss-Prot ID & Description & predicted ec & lift score \\
\hline P388II & Transcription-associated protein I ( $\mathrm{p} 400 \mathrm{kDa}$ component of SAGA). & 2.7.1.137 & 523.71 \\
\hline P23202 & URE2 protein. & 2.5.1.18 & 523.71 \\
\hline Q007I7 & Putative sterigmatocystin biosynthesis protein stcT. & 2.5.1.18 & 523.71 \\
\hline Q6BM74 & URE2 protein. & 2.5.1.18 & 523.71 \\
\hline Q7LLZ8 & URE2 protein. & 2.5.1.18 & 523.71 \\
\hline Q8NJR4 & URE2 protein. & 2.5.1.18 & 523.71 \\
\hline Q8NJR5 & URE2 protein. & 2.5.1.18 & 523.71 \\
\hline Q96WL3 & URE2 protein. & 2.5.1.18 & 523.71 \\
\hline Q96X43 & URE2 protein. & 2.5.1.18 & 523.71 \\
\hline Q96X44 & URE2 protein. & 2.5.1.18 & 523.71 \\
\hline P43589 & Hypothetical 52.2 kDa protein in MPRI-GCN20 intergenic region. & 3.1 .2 .15 & 122.2 \\
\hline O42908 & Hypothetical protein CII9.I7 in chromosome II. & 3.4.24.64 & 192.95 \\
\hline Q10068 & Hypothetical protein $\mathrm{C} 3 \mathrm{HI} .02 \mathrm{c}$ in chromosome $\mathrm{I}$. & 3.4.24.64 & 192.95 \\
\hline Q12496 & Hypothetical I I 8.4 kDa protein in WRSI-PKH2 intergenic region. & 3.4.24.64 & 192.95 \\
\hline P39994 & Hypothetical $61.3 \mathrm{kDa}$ protein in URA3-MMS2I intergenic region. & 4.I.I.I & 261.86 \\
\hline P43546 & Hypothetical 16.6 kDa protein in THI5-AGP3 intergenic region. & I.I.I.- & 112.42 \\
\hline P38169 & Hypothetical $52.4 \mathrm{kDa}$ protein in ATPI-ROX3 intergenic region precursor. & I.14.99.7 & 523.71 \\
\hline PI0662 & Mitochondrial $40 \mathrm{~S}$ ribosomal protein MRPI. & I.I5.I.I & 107.82 \\
\hline P47I4I & Hypothetical $30.2 \mathrm{kDa}$ protein in YUHI-URA8 intergenic region. & I.I5.I.I & 107.82 \\
\hline P53109 & Hypothetical 65.8 kDa protein in SUTI-RCKI intergenic region. & I.16.1.7 & 407.33 \\
\hline P36168 & Hypothetical I37.5 kDa protein in MPLI-PPCI intergenic region. & I.2.1.3 & 174.57 \\
\hline P38992 & SUR2 protein (Syringomycin response protein 2). & 1.3.3.- & 305.5 \\
\hline P36168 & Hypothetical I37.5 kDa protein in MPLI-PPCI intergenic region. & I.5.1.12 & 174.57 \\
\hline P402I5 & Hypothetical 62.8 kDa protein in RPSI6A-TIF34 intergenic region. & 1.8.1.9 & 111.09 \\
\hline P52923 & Hypothetical $41.3 \mathrm{kDa}$ protein in HXTI7-COSI0 intergenic region. & 1.8.1.9 & 111.09 \\
\hline PI4908 & $\begin{array}{l}\text { Mitochondrial replication protein MTFI (Mitochondrial transcription factor mtTFB) } \\
\text { (RFI023) (Mitochondrial specificity factor). }\end{array}$ & 2.1.1.- & 203.67 \\
\hline P87250 & $\begin{array}{l}\text { Mitochondrial replication protein MTFI (Mitochondrial transcription factor } \\
\text { MTTFB). }\end{array}$ & 2.I.I.- & 203.67 \\
\hline
\end{tabular}

\section{Evaluation of the candidate rules}

The criterion satisfied rules were stored in the MySQL database for further evaluation. The testing dataset was used to evaluate the candidate rules governing the enzyme domain composition. Each test datum (separated by commas) was treated as a single string and matched with the set of rules (also separated by commas and treated as a single string) to find the corresponding EC class. The precision of EC class matching (testing dataset to rules set) and the confidence were evaluated using the following equations as given by Kretschmann et al. [7].

$P=$ precision $=\frac{T P}{T P+F P}$

confidence $=\frac{P+\frac{z^{2}}{2 n}-z * \sqrt{\frac{P}{n}-\frac{P^{2}}{n}+\frac{z^{2}}{4 n^{2}}}}{1+\frac{z^{2}}{n}}$ where TP represents the "True Positives" and FP represents the "False Positives" and $z$ is a constant, 1.96 (for 95\% confidence).

\section{Authors' contributions}

SHC implemented the computational approach, performed the analysis and drafted the manuscript. CCC and GYF participated in the design of this study. THL participated in the design of this study, interpreted the results, and wrote the manuscript. All authors read and approved the final manuscript.

\section{Additional material}

\section{Additional File 1}

All rules generated from the fungus training dataset.

Click here for file

[http://www.biomedcentral.com/content/supplementary/1471-

2105-7-304-S1.doc]

$n=T P+F P$ 


\section{Acknowledgements}

We would like to thank anonymous referees for useful comments and suggestions. This work is financially supported by the grant from the Ministry of Economic Affairs (Project No. 93-EC-17-A-17-R7-0563) to Food Industry Research and Development Institute.

\section{References}

I. Gattiker A, Michoud K, Rivoire C, Auchincloss AH, Coudert E, Lima T, Kersey P, Pagni M, Sigrist CJ, Lachaize C, Veuthey AL, Gasteiger E, Bairoch $A$ : Automated annotation of microbial proteomes in SWISS-PROT. Comput Biol Chem 2003, 27(I):49-58.

2. Andrade MA, Brown NP, Leroy C, Hoersch S, de Daruvar A, Reich C, Franchini A, Tamames J, Valencia A, Ouzounis C, Sander C: Automated genome sequence analysis and annotation. Bioinformatics 1999, I 5(5):391-412.

3. Biswas M, O'Rourke JF, Camon E, Fraser G, Kanapin A, Karavidopoulou Y, Kersey P, Kriventseva E, Mittard V, Mulder N, Phan I, Servant F, Apweiler R: Applications of InterPro in protein annotation and genome analysis. Brief Bioinform 2002, 3(3):285-295.

4. Holm L, Sander C: Parser for Protein-Folding Units. Proteins 1994, I 9(3):256-268.

5. Nagarajan N, Yona G: Automatic prediction of protein domains from sequence information using a hybrid learning system. Bioinformatics 2004, 20(9): I335-1360.

6. Apweiler R, Attwood TK, Bairoch A, Bateman A, Birney E, Biswas M, Bucher P, Cerutti L, Corpet F, Croning MD, Durbin R, Falquet L, Fleischmann W, Gouzy J, Hermjakob H, Hulo N, Jonassen I, Kahn D, Kanapin A, Karavidopoulou Y, Lopez R, Marx B, Mulder NJ, Oinn TM, Pagni M, Servant F, Sigrist C], Zdobnov EM: The InterPro database, an integrated documentation resource for protein families, domains and functional sites. Nucleic Acids Res 200I, 29(I):37-40.

7. Kretschmann E, Fleischmann W, Apweiler R: Automatic rule generation for protein annotation with the C4.5 data mining algorithm applied on SWISS-PROT. Bioinformatics 200I, I 7(10):920-926.

8. Bazzan ALC, Engel PM, Schroeder LF, da Silva SC: Automated annotation of keywords for proteins related to mycoplasmataceae using machine learning techniques. Bioinformatics 2002, I8:S35-S43.

9. Creighton C, Hanash S: Mining gene expression databases for association rules. Bioinformatics 2003, I9(I):79-86.

10. Doddi S, Marathe A, Ravi SS, Torney DC: Discovery of association rules in medical data. Med Inform Internet Med 200I, 26(I):25-33.

II. Yang S, Doolittle RF, Bourne PE: Phylogeny determined by protein domain content. PNAS 2005, 1 02(2):373-378.

12. Phan IQ, Pilbout SF, Fleischmann W, Bairoch A: NEWT, a new taxonomy portal. Nucleic Acids Res 2003, 3 I ( I 3):3822-3823.

13. Witten IH, Frank E: Data Mining: Practical machine learning tools and techniques. 2nd edition. San Francisco: Morgan Kaufmann; 2005.

14. Agrawal R, Imielinski T, Swami A: Mining Association Rules between Sets of Items in Large Databases. Proc of the ACM SIGMOD Conference: 1993 1993:207-216.

15. Agrawal R, Srikant R: Fast Algorithms for Mining Association Rules. Proc of the 20th VLDB Conference: 1994 1994:487-499.

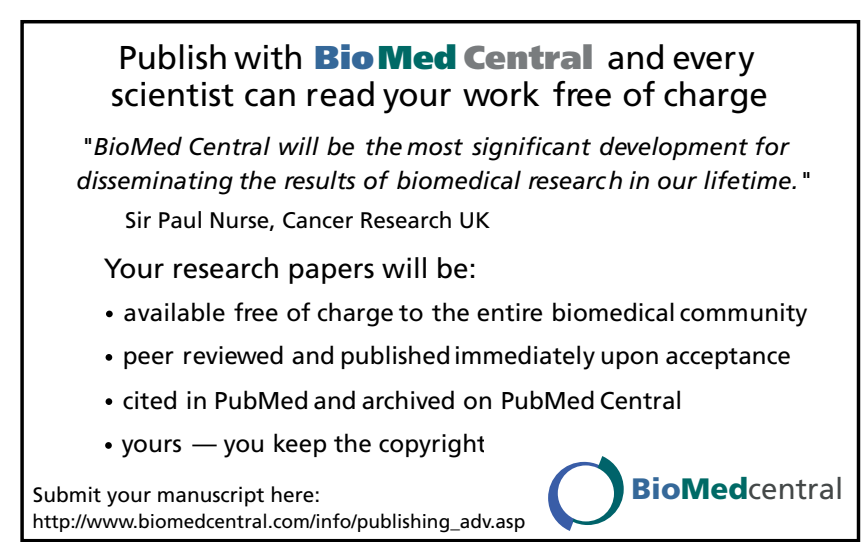

